\title{
Analysis of Data Embedding Techniques for Medical Images
}

\author{
J. Samuel Manoharan, Kezi Selva Vijila, A.Sathesh, and D. Narain Ponraj
}

\begin{abstract}
Data hiding is an age-old technique used to hide data in an image. Data Hiding in Medical images is of great significance, since medical images have to be preserved as such not giving room to any degree of degradation, failing which may lead to wrong diagnosis and treatment. Several attacks are prevalent to hack the data hidden inside the image. Considerable researches are going on in this area to protect the hidden data from unauthorized access. The current work is focused towards studying the behavior of Spatial and Frequency Domain Multiple data embedding techniques for medical images towards noise prone channels enabling the user to select an optimal embedding technique for authentication purposes.
\end{abstract}

Index Terms-Component; medical imaging, authentication, correlation coefficient.

\section{INTRODUCTION}

With the increasing use of Multimedia and related applications over the internet for technological advancements, there is an increasing need for secure data transfer. It is therefore necessary to check and verify the authenticity of the content transmitted or received, to check for any manipulation of data etc., [1]. The most widely used method is the use of Watemarking which can serve allt he above purposes mentioned. At the same time, Watermarking can also be employed to hide or place one content inside another using a visible or invisible methodology for data transfer. A positive approach to the above mentioned method can be put to use in watermarking medical images either ofr data transmission in secrecy or to verify the authenticity of the image. Any data hiding techniques rotate around some critical factors like the robustness of the method, visual imperceptibility of the watermark and embedding capacity.

A General Watermarking system is shown in Fig. 1 where the medical image is taken as the cover image or host image into which one or more number of watermarks of relatively smaller size are embedded using a suitable embedding function. In most of the watermarking techniques developed, the embedding function goes by the way of replacing the host image pixels or coefficients with the watermark image pixels or coefficients.

Pixels are used when the technique is under the spatial

Manuscript received April 9, 2012; revised May 26, 2012.

Kezi Selva Vijila is with Christian College of Enggg, Oddanchatram, South India (e-amil: vijila_ece@yahoo.com)

J. Samuel Manoharan, A. Sathesh, and Narain Ponraj are with the Dept of ECE Karunya University Coimbatore, South India (e-mail: Samuel1530@gmail.com, sathesh_ece@yahooo.com, narainpons@gmail.com) domain and coefficients are used when the technique is a frequency domain technique.

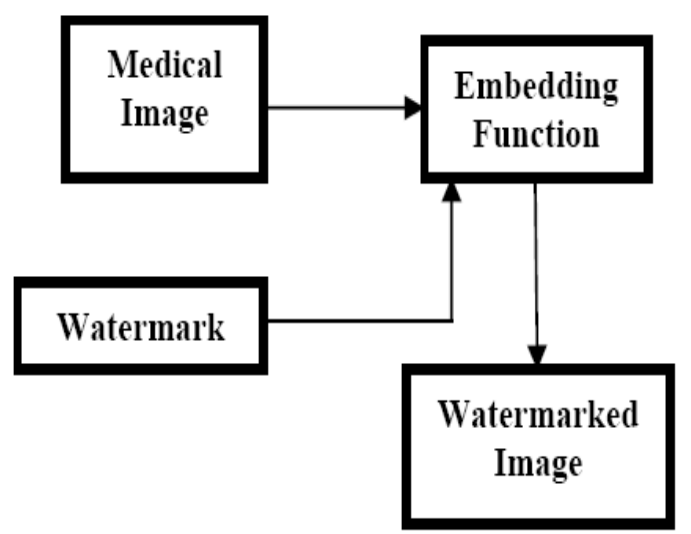

Fig. 1. A General watermarking system

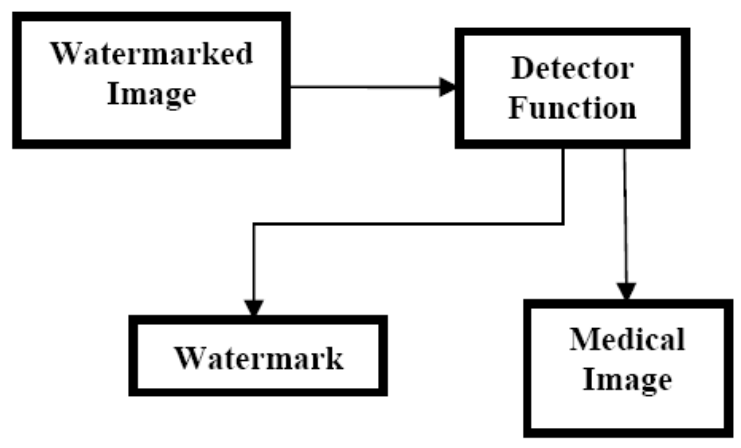

Fig. 2. A general watermarking detection system

Fig. 2 illustrates the flow process of a watermark retrieval or detection system [2] where the watermarked medical image is given to a detector system where suitable and the reverse algorithm of what was applied in the embedding process is used to detect the location of the embedded watermark subsequently followed by its removal thus leaving behind the original cover medical image.

Robustness is a measure of how far the embedded watermark is resistant towards external attacks which is tested by exposing the embedded watermark to various intentional and unintentional image processing operations like adding noise, filtering, rotating etc., Visual Imerceptibility is also an equal parameter which maintained at such a fine degree that no one detects the watermark inside the cover image. Embedding capacity specifies how much of data can be packed into the cover image without affecting the quality of the cover image. The current work is focussed towards embedding a logo or the watermark inside a medical image using techniques in spatial and frequency domain and analyzing its performance towards attacks. 


\section{Methodology}

As mentioned this work involves the useof Embedding Techniques from both Spatial and Frequency Domain and analyzes the performance of these techniques towards attacks. The performance measurement metric used here is the correlation coefficient which is the measure of how far the extracted image resembles the original image after subjection to external attacks. It lies in the interval [0 1$]$. Use of Luminance properties to modify the pixels of the original image is used for spatial domain while Discrete Cosine Transform and Discrete Wavelet Transforms are used for transformations in the Frequency Domain.

\section{A. Spatial Domain Watermarking}

Spatial Domain Watermarking can be achieved using numerous techniques like LSB substitution, Singular Decomposition etc., another alternate method is to separate the color bands in a color image and use any one of the color bands for placing the watermark in which the watermark would be invisible to human eye. However, on separation of the colors [3] the watermark is visible thus causing an essential drawback when imperceptibility is an important criterion in the watermarking process. The data hiding process illustrated here is based on selecting the embedding blocks based on luminance criterion [4] where the host image is converted into $\mathrm{YC}_{\mathrm{b}} \mathrm{C}_{\mathrm{r}}$ images where the luminance containing image alone is taken and modified in accordance with the watermark into chosen blocks whose luminance value is maximum. Retrieval is done using the same separation into components where the block containing the watermark is differenced from the cover image thus getting back the watermark. The retrieved watermark is compared with the original wateramark before embedding and the correlation coefficient is calculated. After the embedding is done, the watermarked image is subjected to a number of intentional and unintentional attacks such as addition of noise, filtering, cropping, rotating and its performance is measure with respect to the correlation coefficient. The correlation coefficient values nearing one indicate a strong embedding algorithm whereas those towards zero indicate a weaker embedding algorithm.
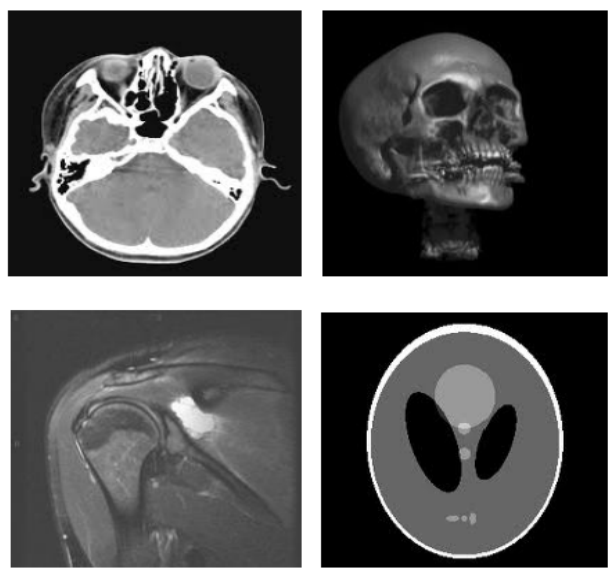

Fig. 3. Cover images used

Fig. 3 and 4 denote the Cover images and the watermarks used respectively. Fig. 5 illustrates the original image on the left hand side and the watermarked medical image on the right hand side. It can be seen that from both the original and watermarked images, the perceptual invisibility is being maintained. These watermarked images are then subjected to external attacks which may be intentional or unintentional and its robustness characteristics are discussed in the later part of this work.

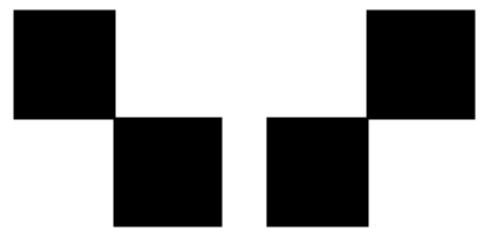

Fig. 4. Watermarks used

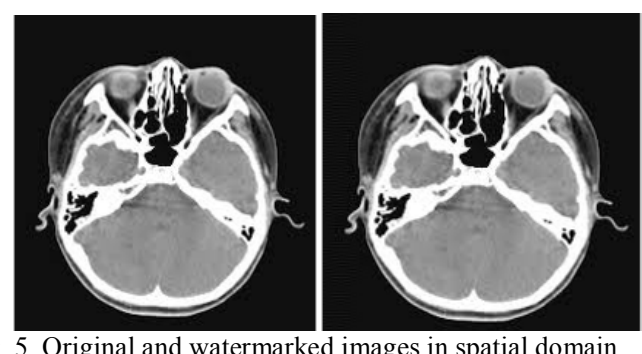

Fig. 5. Original and watermarked images in spatial domain

\section{B. Discrete Cosine Transform}

A Discrete Cosine Transform a sequence of finite data points interms of a sum of cosine functions oscillating at different frequencies. DCTs are important to numerous applications in science and engineering, from lossy compression of audio and images to spectral methods for the numerical solution of partial differential equations. Embedding is achieved by inserting the watermark into a selected set of DCT coefficients [6] [7]. After embedding, the watermark is adapted to the image by exploiting the masking characteristics of the human visual system, thus ensuring the watermark invisibility. Experimental results demonstrate that the watermark is considerably robust to several signal processing techniques, including JPEG compression, addition of Gaussian noise, rotation, and random noise. The host image and watermark are taken and the DCT coefficients of the image and watermarks are obtained using DCT through Block Processing. The Coefficients of the watermarked image are modified as per the equation shown below

$$
\text { Modi_C }\{i, j\}=\beta^{*} C\{i, j\}+\alpha * W\{i, j\}
$$

where $\beta$ is the scaling factor and $\alpha$ is the embedding factor. Finally the inverse DCT is applied to get back the watermarked Image.
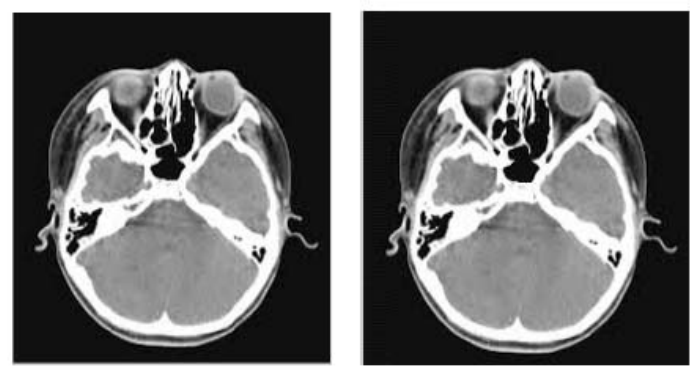

Fig. 6. Original and watermarked images in DCT domain 


\section{Discrete Wavelet Transform}

The host image and watermark is taken and a 3 level DWT is performed on the cover image as well as watemark and the LH or HL bands are chosen for the embedding location [8] - [10]. The DWT coefficients of the data to be hidden are then embedded into these sub bands using the following modification.

$$
\text { Modi_C }\{i, j\}=C\{i, j\}+\alpha * W\{i, j\}
$$

where $C\{i, j\}$ are the host image coefficients $W\{i, j\}$ are the watermark coefficients

$\alpha$ is the embedding factor which is chosen as 3 to provide a tradeoff between invisibility and robustness. For extraction, the watermarked image is subjected to the same three level decomposition using the same wavelet function and on identification of the embedding blocks; the watermark is extracted by differencing with the cover image. The extracted watermark is then checked for its fidelity [11] in terms of the correlation coefficient.
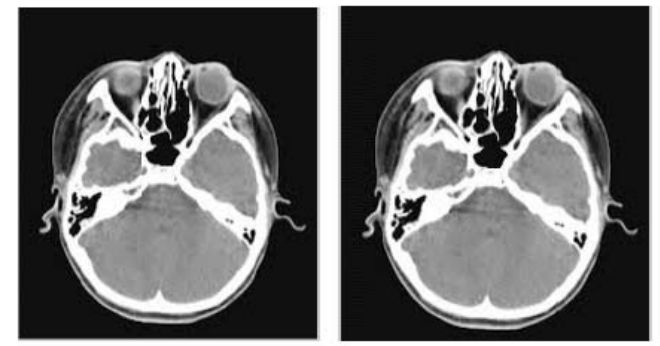

Fig. 7. Original and watermarked images in DWT domain

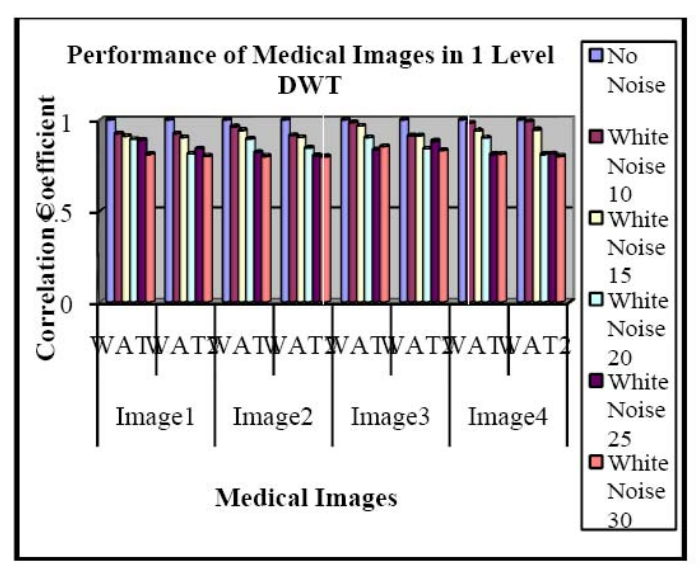

Fig. 8. Performance of the watermarks towards a 1 level DWT

\section{RESULTS AND DISCUSSION}

To begin with 4 Medical Images of size 256 x256 were taken which include a PET Image, Cranial Image, MRI image etc., as shown in Fig 5, and subject to embedding in the spatial as well as frequency domain processing as illustrated previously using more than one number of watermarks. The performance metric is analyzed by subjecting these watermarked images to external noise, cropping, rotation, filtering etc., and its robustness to these attacks is measured in terms of its correlation coefficient. It can be seen that from Fig. that Spatial domain techniques exhibit poor level of tolerance when exposed to intentional and unintentional attacks. Hence, frequency domain techniques seem to be the ideal tool for embedding multiple watermarks in a single image under noisy conditions

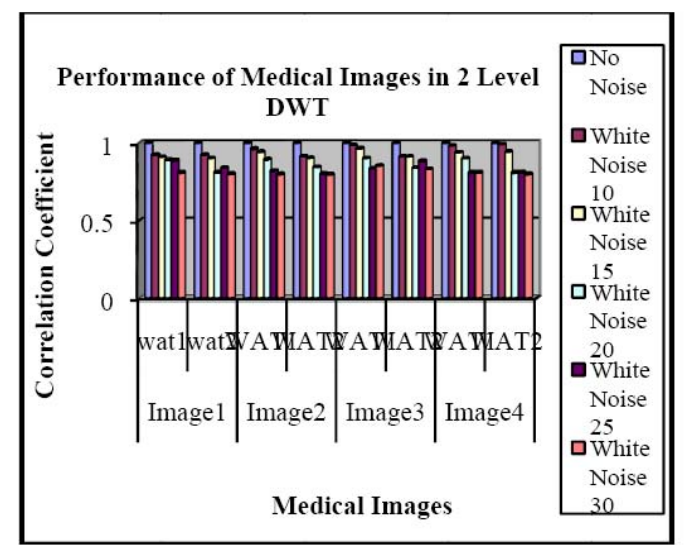

Fig. 9. Performance of the watermarks towards a 2 LEVEL DWT

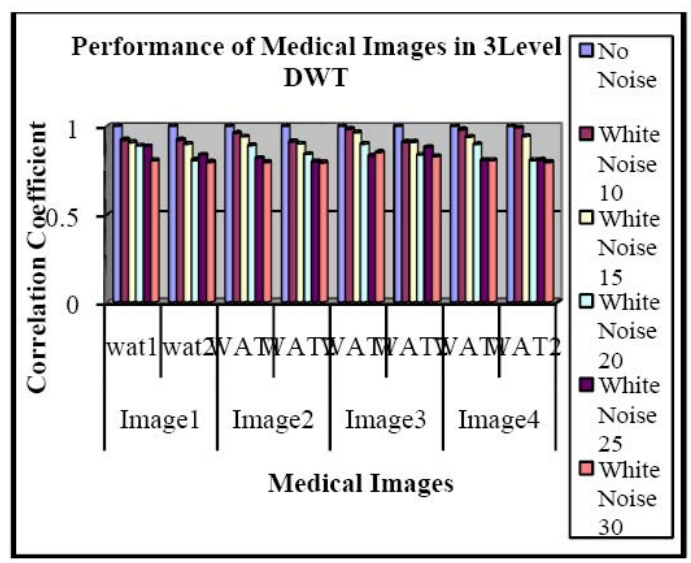

Fig. 10. Performance of the watermarks towards a 3 level DWT

Hence, we have turned our interest as to how frequency domain techniques in DCT and DWT domain behave to noisy conditions. It is once again evident from the results shown that DCT provides good robustness towards channel noise, but does not offer the flexibility of choice of embedding locations relative to the DWT method which offers sparse distributions and is also well suited for images characterized by sharp discontinuities. Extending beyond the scope, we have also explored the characteristics of variation of number of decomposition levels to the detection level of the two watermarks as shown in Figures 8, 9 and 10 for one level, two levels and three levels of DWT decomposition of the original and watermark images.

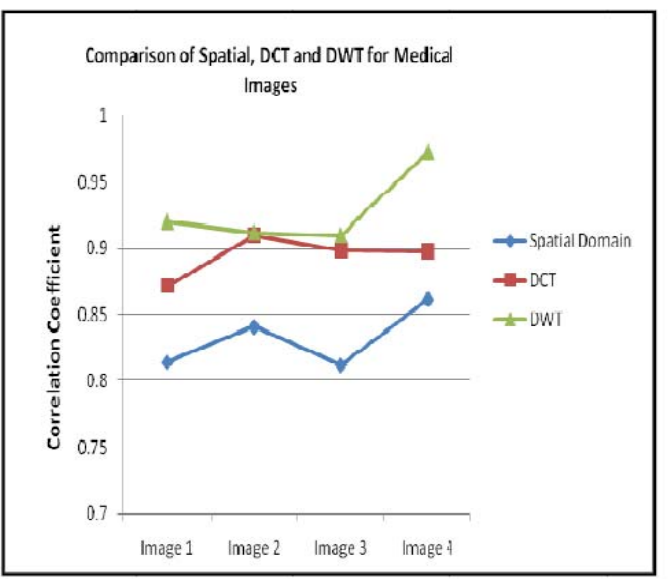

Fig. 11. Performance of the images towards a 1 LEVEL DWT 
From the above work, find a variation of the performance characteristics with changing embedding domains. It can be seen from Figure 11 that spatial domain exhibits a poor tolerance but is found to exhibit a degree of reconstruction factor or fidelity in absence of noise. As mentioned before, DCT domain techniques are quite robust but offer less flexibility in distribution of watermark over the entire image. On the other hand, wavelet based techniques offer good response especially to images with sharp discontinuities which is an essential feature of a medical image. With the above results at hand, it would be a good choice to investigate the behavior of wavelet or DCT based techniques for embedding patient information or the diagnosis report inside the same cover image. It would also provide an insight into the tradeoff between the maximum embedding capacity and the image quality.

\section{REFERENCES}

[1] C. S. Lu, Y. M. Liao, and C. Sze, "Combined Watermarking for Image Authentication and Protection," in Proceedings of the IEEE International Conference on Multimedia and Expo, vol. 3, pp. 1415 1418, 2000.
[2] C. S. Lu and H. Y. M. Liao, "Multipurpose watermarking for image authentication and protection," IEEE Trans. Image Processing, vol. 10, no. 10, pp.1579-1592, 2001

[3] J. P. Gonzalez, "DCT domain Watermarking Techniques: Detector Performance analysis and a new structure," IEEE Transactions on Image Processing, vol. 9, pp. 55 - 68, 2000.

[4] J. Hussein, "Spatial domain Watermarking scheme for color images based on log average luminance," in Journal of Computing, vol. 2 no. $1,2010$.

[5] B. P. Chassery, J. M, and B. Macq, "Geometrically Invariant watermarking using feature points," in IEEE Transactions on Image Processing, vol. 11, no. 9, 2002.

[6] S. P. Mohanty, K. R. Ramakrishnan, and M. S. Kankanhalli, "A DCT Domain Visible Watermarking Technique for Images," by ICME, vol. 2, pp. 1029 - 1032, 2000.

[7] M. Barni, F. Bartolini, V. Cappellini, and A. Piva, "A DCT domain system for robust image watermarks," International Conference on SignalProcessing, vol. 66, no. 3, pp. 357-372, 1998.

[8] A. Lumini and D. Maio, "A Wavelet Based Image watermarking scheme," The International Conference on Information Technology, Coding and Computing (ITCC), pp. 122-127, 2000

[9] Y. Wang, J. F. Doherty, and R. E. V. Dyck, "A Wavelet-Based Watermarking Algorithm for Ownership Verification of Digital Images," IEEE Transactions on Image Processing, vol. 11, no. 2, pp. 77-88, 2002

[10] V. Licks and R. Jordan, "On Digital Image Watermarking Robust to Geometric Transformations," in Proceedings of 2000 International Conference Image Processing (ICIP 2000), vol. 3, pp. 690-693, 2000.

[11] A. A. Haj, "Combined DWT - DCT Digital Image Watermarking," Journal of Computer Science, vol. 3, no.9, pp.740-746, 2007. 